\title{
Adenocarcinoma within anorectal fistulae: different clinicopathological characteristics between Crohn's disease- associated type and the usual type
}

\author{
Makoto Kodama ${ }^{1,2} \cdot$ Daisuke Kobayashi $^{1} \cdot K$ Kuniko lihara ${ }^{2} \cdot K^{2}$ Keiko Abe $^{2} \cdot$ Rikisaburo Sahara $^{3} \cdot$ Motoki Sassa $^{3}$. \\ Tetsuo Yamana ${ }^{3}$ Satomi Furukawa ${ }^{3} \cdot$ Takashi Yao $^{4} \cdot$ Keisuke Uchida $^{5} \cdot$ Tomoki Tamura $^{5} \cdot$ Mariko Negi $^{1}$. \\ Yoshinobu Eishi ${ }^{1}$
}

Received: 13 February 2018 / Revised: 31 May 2018 / Accepted: 6 June 2018 / Published online: 11 September 2018

(c) United States \& Canadian Academy of Pathology 2018

\begin{abstract}
Adenocarcinoma within anorectal fistulae is rare and is sometimes associated with Crohn's disease. Crohn's diseaseassociated adenocarcinoma within anorectal fistulae has a poor prognosis; however, little is known about the clinicopathological differences between Crohn's disease-associated adenocarcinoma within anorectal fistulae and usual adenocarcinoma within anorectal fistulae. We retrospectively searched patients' charts and pathology archives at Tokyo Yamate Medical Center and Tokyo Medical and Dental University Hospital for adenocarcinoma within anorectal fistulae. Clinical and pathological data were collected and immunohistochemical examinations were conducted. Overall survival rate was estimated using the Kaplan-Meier method. Prognostic factors of overall survival were assessed using univariate and multivariate Cox regression analyses. We examined 82 cases of adenocarcinoma within anorectal fistulae. Fifty-nine of 82 cases $(72 \%)$ had usual adenocarcinoma within anorectal fistulae, while the remaining 23 cases (28\%) had Crohn's diseaseassociated adenocarcinoma within anorectal fistulae. Patients with Crohn's disease-associated adenocarcinoma within anorectal fistulae were diagnosed at a younger age and at a more advanced stage than those with usual adenocarcinoma within anorectal fistulae. Macroscopic and histological types were also different between usual adenocarcinoma within anorectal fistulae and Crohn's disease-associated adenocarcinoma within anorectal fistulae. Crohn's disease-associated adenocarcinoma within anorectal fistulae included more ulcerative types and high-grade adenocarcinomas. The rate of lymphovascular invasion was higher in Crohn's disease-associated adenocarcinoma within anorectal fistulae. Immunohistochemically, the expression of E-cadherin, p53, and MUC5AC differed between usual adenocarcinoma within anorectal fistulae and Crohn's disease-associated adenocarcinoma within anorectal fistulae. Patients with Crohn's disease-associated adenocarcinoma within anorectal fistulae exhibited worse overall survival than those with usual adenocarcinoma within anorectal fistulae, and vascular invasion was the strongest significant independent predictor of overall survival in patients with adenocarcinoma within anorectal fistulae. In conclusion, usual adenocarcinoma within anorectal fistulae and Crohn's disease-associated adenocarcinoma within anorectal fistulae have different clinicopathological characteristics and should be considered separate clinical entities.
\end{abstract}

These authors contributed equally: Makoto Kodama, Daisuke

Kobayashi

Daisuke Kobayashi

d-koba.pth1@tmd.ac.jp

1 Department of Human Pathology, Tokyo Medical and Dental University, 1-5-45 Yushima, Bunkyo-ku, Tokyo, Japan

2 Department of Pathology, Tokyo Yamate Medical Center, 3-22-1, Hyakunincho, Shinjuku-ku, Tokyo, Japan
3 Department of Coloproctology Center, Tokyo Yamate Medical Center, 3-22-1, Hyakunincho, Shinjuku-ku, Tokyo, Japan

4 Department of Human Pathology, Juntendo University Graduate School of Medicine, 2-1-1 Hongo, Bunkyo-ku, Tokyo, Japan

5 Division of Surgical Pathology, Tokyo Medical and Dental University Hospital, 1-5-45 Yushima, Bunkyo-ku, Tokyo, Japan 


\section{Introduction}

Adenocarcinoma within anorectal fistulae is a rare tumor and is defined by the World Health Organization (WHO) 2010 classification as a tumor developing in a pre-existing anal sinus or fistula [1]. Carcinoma within anorectal fistulae mainly consists of adenocarcinoma, with mucinous adenocarcinoma being the most frequent. However, there are a small number of squamous cell carcinomas in anorectal fistulae $[1,2]$. Some adenocarcinoma within anorectal fistulae are associated with Crohn's disease [3]. Iesalnieks et al. [4] reported that the outcome of Crohn's diseaseassociated associated adenocarcinoma within anorectal fistulae was poor, based on the clinical examination of six cases of Crohn's disease-associated adenocarcinoma within anorectal fistulae and a systematic review of 65 cases of Crohn's disease-associated adenocarcinoma within anorectal fistulae. However, to our knowledge, there has been no report in the English literature comparing Crohn's diseaseassociated adenocarcinoma within anorectal fistulae with usual adenocarcinoma within anorectal fistulae, which is not associated with Crohn's disease. The present study assessed the difference in clinicopathological characteristics between usual adenocarcinoma within anorectal fistulae and Crohn's disease-associated adenocarcinoma within anorectal fistulae.

\section{Materials and methods}

\section{Case selection}

The present study was approved by the Ethics Committee of Tokyo Yamate Medical Center (216) and Tokyo Medical and Dental University Hospital (M2017-070). Charts and pathology archives were searched for patients who had been diagnosed with adenocarcinoma located in the anorectum from 1999 to 2016 and met the criteria for adenocarcinoma within anorectal fistulae in the WHO 2010 classification [1].

\section{Clinical data collection}

We collected clinical data including sex, age at diagnosis of cancer, duration of anal fistula, survival time, presence of other organ metastasis, dissemination, local recurrence in adenocarcinoma within anorectal fistulae, and duration of Crohn's disease in Crohn's disease-associated adenocarcinoma within anorectal fistulae cases. Some clinical data could not be obtained in all cases, which were as follows: preoperative serum carcinoembyonic antigen (CEA) level, preoperative serum carbohydrate antigen 19-9 (CA19-9) level, and the type of pre-existing anal fistula. Durations of anal fistula and Crohn's disease were defined as the period from the first diagnosis of anal fistula and Crohn's disease to the diagnosis of adenocarcinoma within anorectal fistulae, respectively. The site of metastasis and presence of local recurrence and dissemination were determined on the basis of imaging records and cytological or histological examination.

\section{Pathological data collection}

We examined pathological data of the cases of adenocarcinoma within anorectal fistulae that were surgically treated. Pathological data included macroscopic type, histological type, tumor depth (T stage) [5], and presence of vascular and lymphatic invasion. The macroscopic type of adenocarcinoma within anorectal fistulae was classified into three groups as follows: "endophytic type" that remains within the anal wall and is not exposed to the surface (Fig. 1a, b), "exophytic type" that is exposed to the surface of the anal canal with or without protrusion (Fig. 1c, d), and "ulcerative type" that forms ulceration, damages the anal wall, and resembles Borrmann's Type 2 or 3 disease (Fig. 1e, f). The histological type of adenocarcinoma within anorectal fistulae was classified into tubular adenocarcinoma and mucinous adenocarcinoma. Both adenocarcinomas were further divided into low grade $(>50 \%$ gland forming) (Fig. 2a) and high grade (0-49\% gland forming) (Fig. 2b), according to the WHO classification system [1]. Lymphatic invasion was evaluated using hematoxylin and eosin staining. Vascular invasion was evaluated using Elastica-van Gieson staining. T stage categories were determined according to the TNM classification (Union for International Cancer Control; UICC, 2016) [5]. The tumor stage was also determined according to the TNM classification (UICC, 2016) based on both pathological and clinical data, if available.

\section{Immunohistochemical data}

The immunohistochemical antibodies listed in Table 1 were used in the present study. All tissues were fixed in $10 \%$ formalin after surgical resection and embedded in paraffin. Three micrometer-thick sections were cut from the paraffin tissue block, deparaffinized in xylene, and dehydrated in ethanol. Slides were pretreated for antigen retrieval as indicated in Table 1. All slides were incubated with $3 \%$ hydrogen peroxide to block endogenous peroxidase activity. Primary antibodies were applied as described in Table 1. After washing in phosphate-buffered saline with $0.005 \%$ Tween 20 , slides were incubated with horseradish peroxidase-conjugated anti-rabbit or anti-mouse Ig polymer as a secondary antibody (Envision kit; Dako, Glostrup, Denmark) for $30 \mathrm{~min}$ at room temperature. The slides were treated with a streptavidin-peroxidase reagent and incubated 
Fig. 1 Representative images of macroscopic types of adenocarcinoma within anorectal fistulae. a, b The typical macroscopic appearance of the "endophytic type" and the cut surface which was cut along the yellow line. Arrow heads show endophytic tumor. c, d The typical macroscopic appearance of the "exophytic" and the cut surface, which was cut along the yellow line. Arrow head shows exophytic tumor. e, f The typical macroscopic appearance of the "ulcerative type" and the cut surface, which was cut along the yellow line. Arrow heads show ulcerative tumor

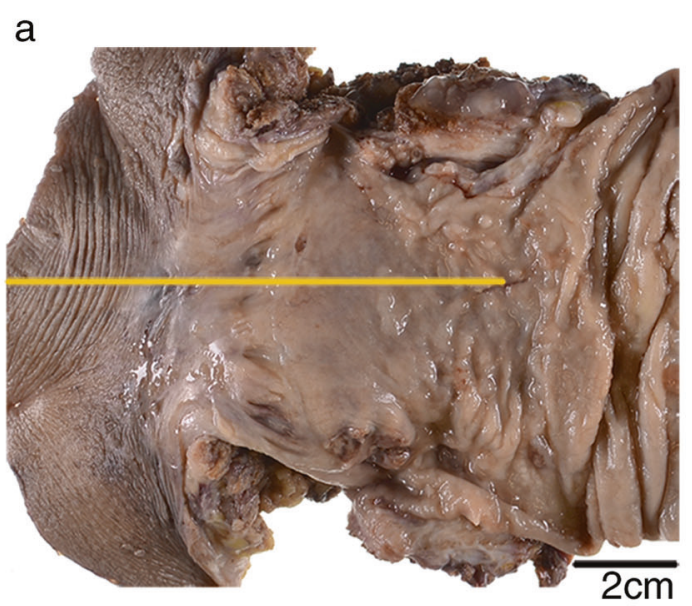

b
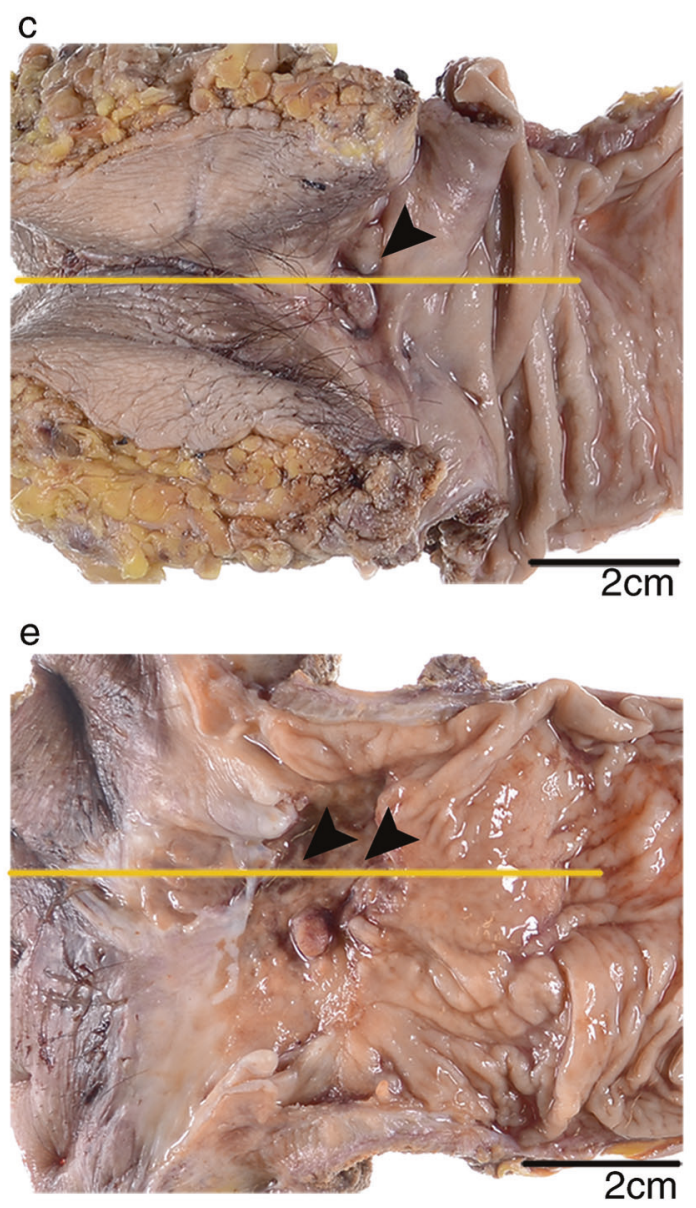

d

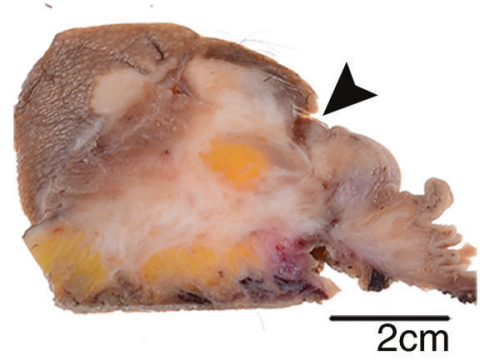

$f$
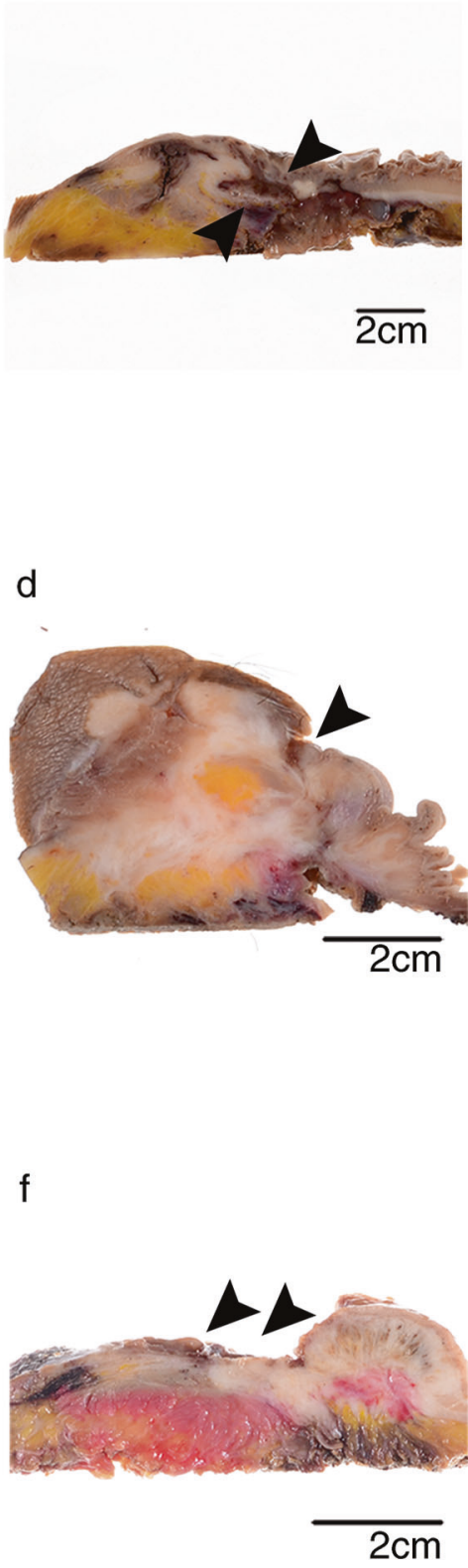

in phosphate-buffered saline with $0.005 \%$ Tween 20 . Visualization was achieved by incubating the slides for $10 \mathrm{~min}$ in a solution of $0.1 \mathrm{M}$ Tris- $\mathrm{HCl}$ containing $0.05 \%$ 3-3'-diaminobenzidine, $0.04 \%$ nickel chloride, and $0.0075 \% \mathrm{H}_{2} \mathrm{O}_{2}$, followed by counterstaining with Mayer's hematoxylin.

Staining positivity for each of the antibodies was evaluated as described in Table 1. The immunohistochemical results of MUC1, MUC2, MUC5AC, MUC6, CK7, CK20,
CDX2, and p53 were considered positive when more than $10 \%$ of cells were stained [6, 7]. Ki-67 and p16 staining was considered positive when more than $50 \%$ of cells were stained [8].

E-cadherin was evaluated by both staining intensity and area, according to a previous report [9]. $\beta$-catenin expression was accepted to be aberrant when there was a lack of membrane and nuclear ectopic localization. Mismatch repair protein expression was evaluated with mutL homolog 
Fig. 2 Typical histological findings and immunohistochemical pattern of low grade (a, c, e) and high grade $(\mathbf{b}, \mathbf{d}, \mathbf{e})$ mucinous adenocarcinoma. $\mathbf{a}, \mathbf{b}$

Hematoxylin and eosin stain. c, $\mathbf{d}$ Immunohistochemical staining of E-cadherin. e, f Immunohistochemical staining of p53. $\mathbf{g}, \mathbf{h}$ Immunohistochemical staining of MUC5AC. $(\mathbf{a}-\mathbf{h} \times 400)$

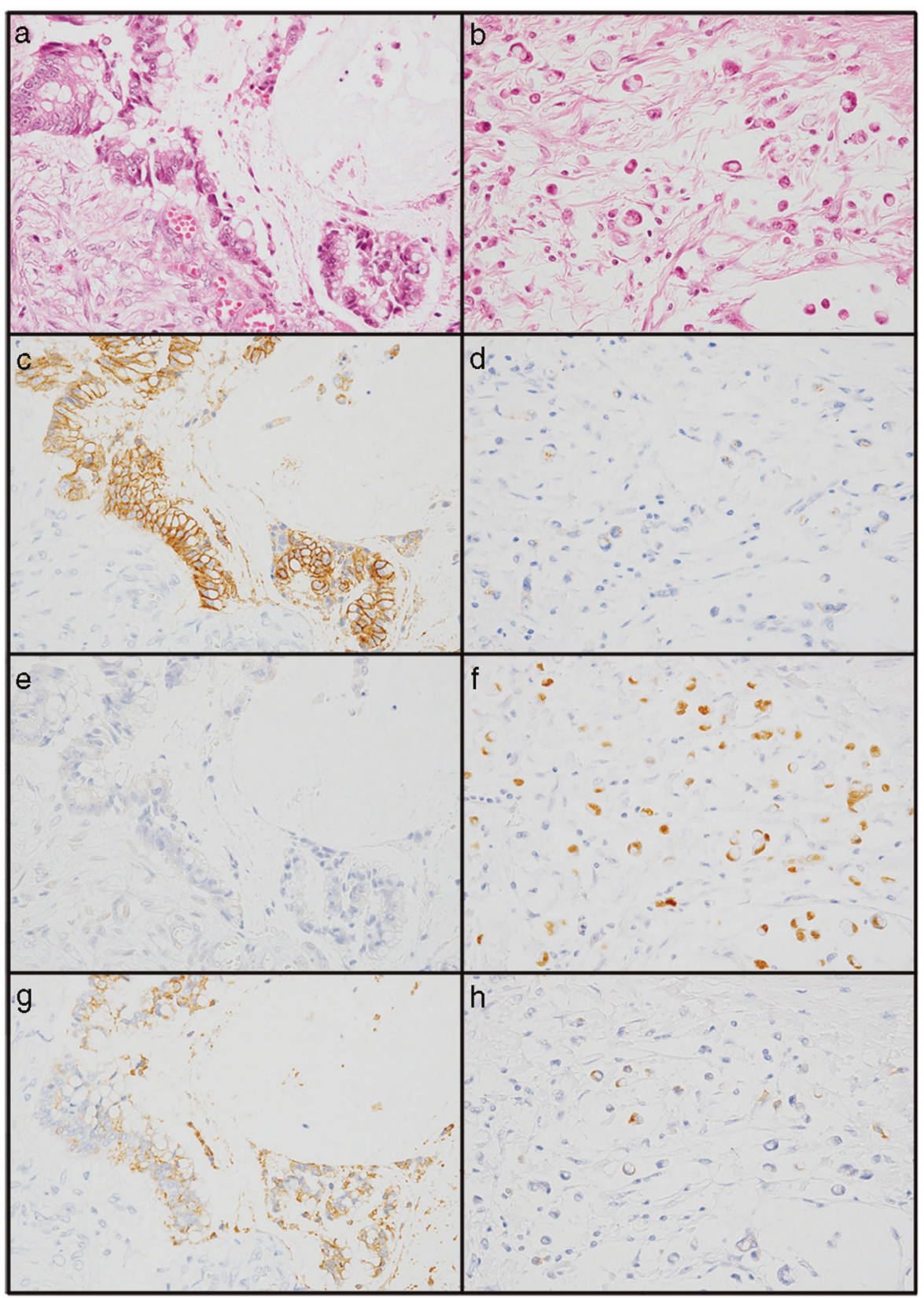

1, PMS1 homolog 2, mutS protein homolog 2, and mutS protein homolog 6 . The proteins were considered lost when nuclear staining was completely absent.

\section{Statistical analyses}

All analyses were two-sided and $p<0.05$ was considered significant. The chi-squared exact test was used to determine the association between different variables. Overall survival curves were obtained using the Kaplan-Meier method and compared using the log-rank test and generalized Wilcoxon test. Overall survival was determined as the time from surgery to death from any cause or last known follow-up. Multivariate Cox regression analysis with stepwise selection was used to examine independent prognostic factors associated with overall survival. All statistical analyses and graphical representations were performed using SPSS 16.0 (SPSS Inc.; Chicago, USA) and GraphPad Prism 6 (GraphPad Software, San Diego, USA) software.

\section{Results}

\section{Case selection}

We found 83 cases of adenocarcinoma within anorectal fistulae at two hospitals during the designated period, as well as five cases of squamous cell carcinoma within 
Table 1 Panel of antibodies for immunohistochemical analysis

\begin{tabular}{|c|c|c|c|c|c|c|}
\hline Antibody & Dilution & Clone & Manufacturer & Pretreatment & Incubation time & Staining evaluation \\
\hline CK7 & $1: 20$ & M7018 & DAKO & Microwave $\dagger$ & $60 \min$ & Cytoplasmic \\
\hline CK20 & $1: 20$ & M7019 & DAKO & Protease & $20 \min$ & Cytoplasmic \\
\hline $\mathrm{CDX} 2$ & $1: 50$ & M3636 & DAKO & Microwave $\dagger$ & $60 \min$ & Nuclear \\
\hline MUC1 & $1: 1000$ & MA552 & Leica Biosytems & None & $60 \mathrm{~min}$ & Cytoplasmic \\
\hline MUC2 & $1: 100$ & Ccp58 & Leica Biosytems & Autoclave & Overnight & Cytoplasmic \\
\hline MUC5AC & $1: 1600$ & CLH-2 & Leica Biosytems & Autoclave: & Overnight & Cytoplasmic \\
\hline MUC6 & $1: 50$ & CLH-5 & Leica Biosytems & Autoclave $\$$ & Overnight & Cytoplasmic \\
\hline p16 & Pre-diluted & 9517 & CINtec & Microwave $\dagger$ & $60 \min$ & Nuclear \\
\hline p53 & $1: 1000$ & DO7 & Leica Biosytems & Autoclave $\$$ & Overnight & Nuclear \\
\hline Ki-67 & $1: 800$ & M7240 & DAKO & Autoclave $\$$ & Overnight & Nuclear \\
\hline E-cadherin & $1: 100$ & M3612 & DAKO & Autoclave & Overnight & Membranous \\
\hline$\beta$-catenin & $1: 50$ & M3539 & DAKO & Microwave $\dagger$ & $60 \mathrm{~min}$ & Nuclear \\
\hline MLH1 & $1: 100$ & ES05 & DAKO & Autoclave & Overnight & Nuclear \\
\hline PMS2 & $1: 3000$ & 556415 & BD Bioscience & Autoclave: & Overnight & Nuclear \\
\hline MSH2 & $1: 10000$ & 556349 & BD Bioscience & Autoclaveł & Overnight & Nuclear \\
\hline MSH6 & $1: 10$ & $08-1374$ & Zymed & Autoclave & Overnight & Nuclear \\
\hline
\end{tabular}

$\dagger 97^{\circ} \mathrm{C}, 40 \mathrm{~min},{ }^{\ddagger} 120^{\circ} \mathrm{C}, 20 \mathrm{~min}$ anorectal fistulae, although these five cases were excluded. Fifty-nine of the 83 cases ( $71 \%$ ) had usual adenocarcinoma within anorectal fistulae, 23 cases (28\%) had Crohn's disease-associated adenocarcinoma within anorectal fistulae, and one case $(1 \%)$ was associated with ulcerative colitis. We excluded the case with ulcerative colitis, and in total, 82 cases of adenocarcinoma within anorectal fistulae were examined. Seventy-five patients were treated surgically and were examined pathologically. Seven patients were treated non-surgically because six were too far advanced to perform surgery, and one patient refused to undergo surgery.

\section{Clinical data}

The clinical data for usual adenocarcinoma within anorectal fistulae and Crohn's disease-associated adenocarcinoma within anorectal fistulae are shown in Table 2. Male predilection was detected in both usual adenocarcinoma within anorectal fistulae and Crohn's disease-associated adenocarcinoma within anorectal fistulae groups, and the tendency was more pronounced in the usual adenocarcinoma within anorectal fistulae group $(p=0.0004)$. Crohn's disease-associated adenocarcinoma within anorectal fistulae was diagnosed at an average age of 40 years, which was significantly younger than the age of diagnosis for usual adenocarcinoma within anorectal fistulae $(p<$ $0.0001)$. The mean duration of anal fistulae was 12.5 $(<1-50)$ years in the usual adenocarcinoma within anorectal fistulae group and $9.1(<1-29)$ years in the Crohn's disease-associated adenocarcinoma within anorectal fistulae group. There were a few cases with a very short period of pre-existing anorectal fistulae; however, the duration was not defined according to the WHO 2010 classification. The mean duration of Crohn's disease in Crohn's disease-associated adenocarcinoma within anorectal fistulae patients was 19.3 (5-42) years. Some clinical data could not be obtained in all cases and are therefore described below and not listed in Table 2. An elevated serum CEA level was detected in 26 out of 47 cases $(55 \%)$ with usual adenocarcinoma within anorectal fistulae and in 13 out of 17 cases (77\%) with Crohn's diseaseassociated adenocarcinoma within anorectal fistulae. An elevated serum CA-19-9 level was detected in 15 out of 47 cases $(32 \%)$ with usual adenocarcinoma within anorectal fistulae and 7 out of 16 cases (44\%) with Crohn's disease-associated adenocarcinoma within anorectal fistulae. The elevation of at least one of CEA or CA-19-9 was detected in 30 of 47 cases $(64 \%)$ with usual adenocarcinoma within anorectal fistulae and in 15 of 17 cases (88\%) with Crohn's disease-associated adenocarcinoma within anorectal fistulae. The types of pre-existing anal fistula were as follows: of 44 usual adenocarcinoma within anorectal fistulae cases, $10(23 \%)$ were the intrasphincteric type, $16(36 \%)$ passed through the ischiorectal fossa, $13(30 \%)$ passed through the pelvic rectal fossa, and $5(11 \%)$ were unclassifiable because of their complexity. Among six Crohn's disease-associated adenocarcinoma within anorectal fistulae cases, one case $(17 \%)$ was the intersphincteric type, one case (17\%) passed through the pelvic rectal fossa, and four cases $(67 \%)$ were unclassifiable because of their complexity. 


\section{Pathological data}

The pathological data for usual adenocarcinoma within anorectal fistulae and Crohn's disease-associated adenocarcinoma within anorectal fistulae groups are presented in Table 3. The macroscopic type in usual adenocarcinoma within anorectal fistulae was frequently the endophytic type or the exophytic type, whereas in Crohn's diseaseassociated adenocarcinoma within anorectal fistulae the most frequent was the ulcerative type. Mucinous adenocarcinoma was the most common histological type in both categories of adenocarcinoma within anorectal fistulae, unlike conventional colorectal carcinoma. High-grade adenocarcinoma was only detected in mucinous carcinoma and the rate of high-grade adenocarcinoma was significantly higher in Crohn's disease-associated adenocarcinoma within anorectal fistulae than in usual adenocarcinoma within anorectal fistulae $(p<0.0001)$. Both vascular and lymphatic invasion were observed at significantly higher rates in Crohn's disease-associated adenocarcinoma within anorectal fistulae than in usual adenocarcinoma within anorectal fistulae (vascular invasion; $p=0.0010 /$ lymphatic invasion; $p=0.0216$ ). In patients with Crohn's diseaseassociated adenocarcinoma within anorectal fistulae, active Crohn's disease inflammation was observed in 6 of 17 cases $(35 \%)$ in the anal canal or in the rectum adjacent to the anal canal, and epithelioid cell granulomas were detected in 5 of 17 cases $(29 \%)$.

\section{Tumor stage}

The tumor progression stage in usual adenocarcinoma within anorectal fistulae and Crohn's disease-associated adenocarcinoma within anorectal fistulae is shown in Table 4. Stage II was the most frequent in both usual adenocarcinoma within anorectal fistulae and Crohn's diseaseassociated adenocarcinoma within anorectal fistulae. Unlike usual adenocarcinoma within anorectal fistulae, stage IV was the second most common in Crohn's disease-associated adenocarcinoma within anorectal fistulae and there was a significant difference in tumor staging between the two types of adenocarcinoma within anorectal fistulae $(p=$ 0.0037).

\section{Immunohistochemical data}

Immunohistochemical data for usual adenocarcinoma within anorectal fistulae and Crohn's disease-associated adenocarcinoma within anorectal fistulae are presented in Table 5. There was a significant difference in the expression levels of only three proteins (E-cadherin, p53, and MUC5AC) among the 17 studied between the two types of adenocarcinoma within anorectal fistulae. E-cadherin is a

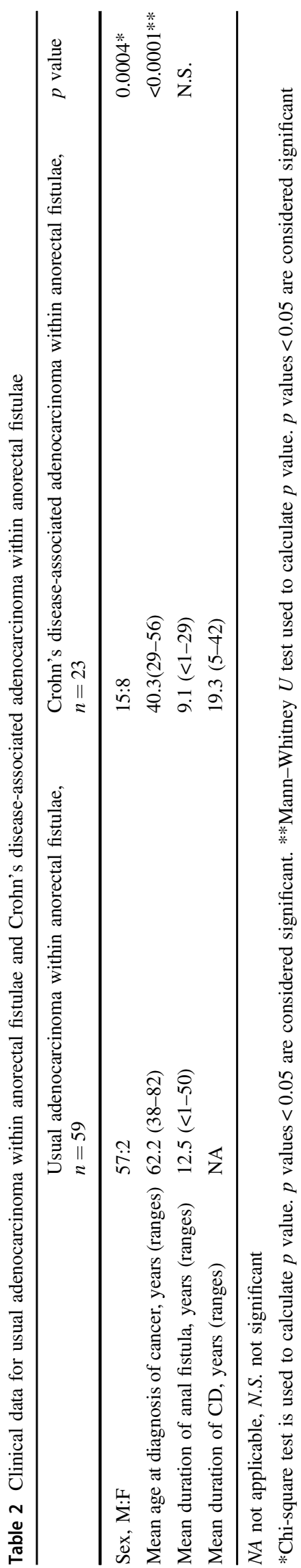


Table 3 Pathological data for usual adenocarcinoma within anorectal fistulae and Crohn's disease-associated adenocarcinoma within anorectal fistulae

\begin{tabular}{|c|c|c|c|}
\hline & \multicolumn{2}{|l|}{ Number of cases $(\%)$} & \multirow[t]{2}{*}{$p$ value* } \\
\hline & $\begin{array}{l}\text { Usual adenocarcinoma } \\
\text { within anorectal fistulae, } \\
n=58\end{array}$ & $\begin{array}{l}\text { Crohn's disease-associated } \\
\text { adenocarcinoma within anorectal } \\
\text { fistulae, } n=17\end{array}$ & \\
\hline Macroscopic type & & & $<0.0001$ \\
\hline Extraluminal type & $27(47)$ & $0(0)$ & \\
\hline Exposed type & $22(38)$ & $4(24)$ & \\
\hline Ulcerative type & $9(16)$ & $13(77)$ & \\
\hline Histological type & & & $<0.0001$ \\
\hline $\begin{array}{l}\text { Tubular adenocarcinoma: } \\
\text { low grade }\end{array}$ & $12(21)$ & $5(29)$ & \\
\hline $\begin{array}{l}\text { Tubular adenocarcinoma: } \\
\text { high grade }\end{array}$ & $0(0)$ & $0(0)$ & \\
\hline $\begin{array}{l}\text { Mucinous carcinoma: low } \\
\text { grade }\end{array}$ & $45(78)$ & $6(35)$ & \\
\hline $\begin{array}{l}\text { Mucinous carcinoma: } \\
\text { high grade }\end{array}$ & $1(2)$ & $6(35)$ & \\
\hline T stage & & & N.S. \\
\hline $\mathrm{T} 1$ & $0(0)$ & $0(0)$ & \\
\hline $\mathrm{T} 2$ & $6(10)$ & $1(6)$ & \\
\hline $\mathrm{T} 3$ & $45(78)$ & $9(53)$ & \\
\hline $\mathrm{T} 4$ & $3(5)$ & $7(41)$ & \\
\hline Vascular invasion & $25(43)$ & $15(88)$ & 0.0010 \\
\hline Lymphatic invasion & $26(45)$ & $13(77)$ & 0.0216 \\
\hline
\end{tabular}

N.S. not significant

*Chi-square test is used to calculate $p$ value. $p$ values $<0.05$ are considered significant

\begin{tabular}{llll}
\hline & \multicolumn{2}{l}{ Number of cases $(\%)$} & $p$ value* \\
\cline { 2 - 3 } & $\begin{array}{l}\text { Usual adenocarcinoma within } \\
\text { anorectal fistulae, } n=59\end{array}$ & $\begin{array}{l}\text { Crohn's disease-associated adenocarcinoma } \\
\text { within anorectal fistulae, } n=23\end{array}$ & 0.0037 \\
\hline Stage & & $1(4)$ & \\
Stage I & $5(9)$ & $12(52)$ & \\
Stage II & $45(76)$ & $4(17)$ & $6(26)$ \\
Stage III & $8(14)$ & $1(2)$ & \\
Stage IV & $1(2)$ &
\end{tabular}

*Chi-square test is used to calculate $p$ value. $p$ values $<0.05$ are considered significant
Table 4 Tumor stage for usual adenocarcinoma within anorectal fistula and Crohn's disease-associated adenocarcinoma within anorectal fistulae associated adenocarcinoma within anorectal fistulae (Fig. 2g, h). The proportions of CK7/CK20 expression patterns were almost the same between usual adenocarcinoma within anorectal fistulae and Crohn's diseaseassociated adenocarcinoma within anorectal fistulae. The predominant pattern was double positive staining, and a normal colorectal pattern (CK7 negative/CK20 positive) was detected in only one third of adenocarcinoma within anorectal fistulae. Loss of mismatch repair protein expression was detected in only one case of usual adenocarcinoma within anorectal fistulae among the four mismatch repair proteins examined in the present study, and the loss was only for MSH6 expression. 
Table 5 Immunohistochemical data for usual adenocarcinoma within anorectal fistulae and Crohn's disease-associated adenocarcinoma within anorectal fistulae

\begin{tabular}{|c|c|c|c|}
\hline & \multicolumn{2}{|l|}{ Number of specimens (\%) } & \multirow[t]{2}{*}{$p$ value* } \\
\hline & $\begin{array}{l}\text { Usual adenocarcinoma within } \\
\text { anorectal fistulae, } n=58\end{array}$ & $\begin{array}{l}\text { Crohn's disease-associated } \\
\text { adenocarcinoma within anorectal fistulae, } \\
n=17\end{array}$ & \\
\hline E-cadherin & $46(79)$ & $4(24)$ & $<0.0001$ \\
\hline P16 & $14(24)$ & $5(29)$ & N.S. \\
\hline P53 & $25(43)$ & $15(88)$ & 0.0017 \\
\hline Ki-67 & $10(17)$ & $6(35)$ & N.S. \\
\hline $\begin{array}{l}\beta \text {-catenin } \\
\text { aberrant }\end{array}$ & $4(7)$ & $1(6)$ & N.S. \\
\hline MMR loss & $1(2)$ & $0(0)$ & N.S. \\
\hline MUC1 & $1(2)$ & $2(12)$ & N.S. \\
\hline MUC2 & $56(97)$ & $16(94)$ & N.S. \\
\hline MUC5AC & $42(72)$ & $5(29)$ & 0.0002 \\
\hline MUC6 & $5(9)$ & $2(12)$ & N.S. \\
\hline $\mathrm{CK} 7 / \mathrm{CK} 20$ & & & N.S. \\
\hline $\begin{array}{l}\text { Positive/ } \\
\text { positive }\end{array}$ & $34(59)$ & $10(59)$ & \\
\hline $\begin{array}{l}\text { Positive/ } \\
\text { negative }\end{array}$ & $3(5)$ & $1(6)$ & \\
\hline $\begin{array}{l}\text { Negative/ } \\
\text { positive }\end{array}$ & $19(33)$ & $6(35)$ & \\
\hline $\begin{array}{l}\text { Negative/ } \\
\text { negative }\end{array}$ & $2(3)$ & $0(0)$ & \\
\hline CDX2 & 54 (93) & $13(77)$ & N.S. \\
\hline
\end{tabular}

N.S. not significant

*Chi-square test is used to calculate $p$ value. $p$ values $<0.05$ are considered significant
Fig. 3 Kaplan-Meier analysis of overall survival (a) in all patients with usual adenocarcinoma within anorectal fistulae and Crohn's disease-associated adenocarcinoma within anorectal fistulae $(p<0.0001)$, and $\mathbf{b}$ in stage II patients with usual adenocarcinoma within anorectal fistulae and Crohn's disease-associated adenocarcinoma within anorectal fistulae $(p=0.0167)$

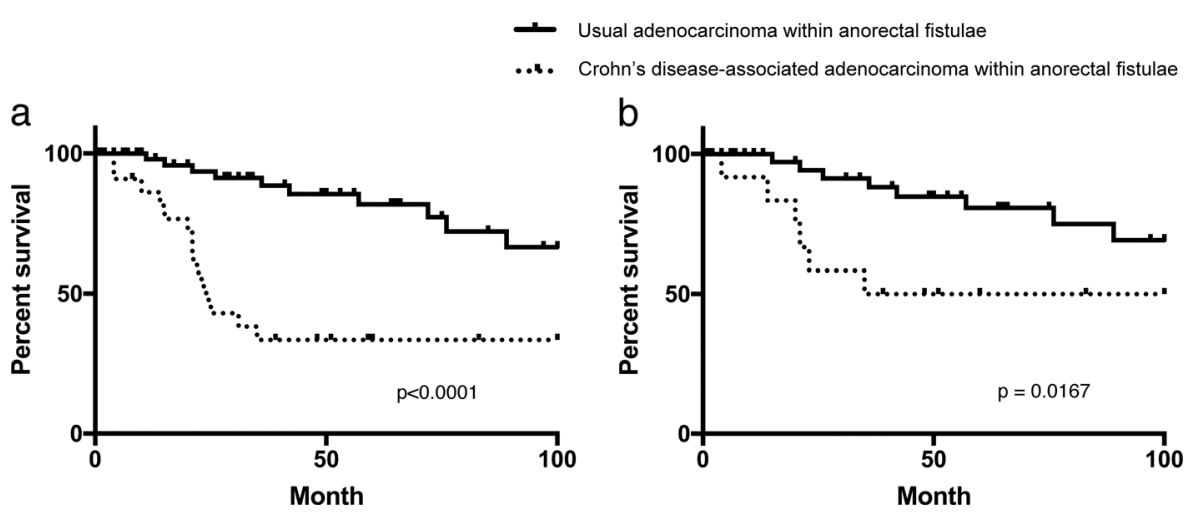

\section{Survival analysis}

The average follow-up period for all cases of adenocarcinoma within anorectal fistulae in the present study was 43.3 months. As shown in Fig. 3a, there was a significant difference in overall survival between all-stage patients with usual adenocarcinoma within anorectal fistulae and those with Crohn's disease-associated adenocarcinoma within anorectal fistulae (overall survival: log-rank test $/ p<$ 0.0001). Furthermore, we compared overall survival between usual adenocarcinoma within anorectal fistulae and
Crohn's disease-associated adenocarcinoma within anorectal fistulae in stage II, which was the most common stage in both types of adenocarcinoma within anorectal fistulae. As shown in Fig. 3b, there was also a significant difference in overall survival with stage II disease (overall survival: log-rank test $/ p=0.0167$ ).

\section{Cancer spread}

Rates of metastasis, dissemination, and local recurrence of usual adenocarcinoma within anorectal fistulae and Crohn's 
Table 6 Metastasis, dissemination, and local recurrence of usual adenocarcinoma within anorectal fistulae and Crohn's disease-associated adenocarcinoma within anorectal fistulae

\begin{tabular}{|c|c|c|c|}
\hline & \multicolumn{2}{|l|}{ Number of cases (\%) } & \multirow[t]{2}{*}{$p$ value* } \\
\hline & $\begin{array}{l}\text { Usual adenocarcinoma within } \\
\text { anorectal fistulae, } n=59\end{array}$ & $\begin{array}{l}\text { Crohn's disease-associated } \\
\text { adenocarcinoma within anorectal } \\
\text { fistulae, } n=23\end{array}$ & \\
\hline $\begin{array}{l}\text { Lymph node } \\
\text { metastasis }\end{array}$ & $7(12)$ & $5(22)$ & N.S. \\
\hline $\begin{array}{l}\text { Other organ } \\
\text { metastasis }\end{array}$ & $7(12)$ & $12(53)$ & 0.0001 \\
\hline At diagnosis & 1 & 4 & \\
\hline Postoperative & 6 & 8 & \\
\hline Dissemination & $2(3)$ & $2(9)$ & N.S. \\
\hline At diagnosis & 0 & 2 & \\
\hline Postoperative & 2 & 0 & \\
\hline Local recurrence & $7(12)$ & $3(13)$ & N.S. \\
\hline
\end{tabular}

N.S. not significant

*Chi-square test is used to calculate $p$ value. $p$ values $<0.05$ are considered significant disease-associated adenocarcinoma within anorectal fistulae are shown in Table 6. Although there was a significant difference in lymphatic invasion between the two types of adenocarcinoma within anorectal fistulae, the rate of lymph node metastasis was not significantly different between the two types. Other organ metastasis was detected in 7 of 59 cases (12\%) with usual adenocarcinoma within anorectal fistulae and in 12 of 23 cases (53\%) with Crohn's diseaseassociated adenocarcinoma within anorectal fistulae; the rate was significantly greater in patients with Crohn's disease-associated adenocarcinoma within anorectal fistulae. In one case with usual adenocarcinoma within anorectal fistulae and four cases with Crohn's disease-associated adenocarcinoma within anorectal fistulae, other organ metastasis was detected at the diagnosis of adenocarcinoma within anorectal fistulae. The lung was the most common metastatic organ in usual adenocarcinoma within anorectal fistulae (five cases, 71\%), and the others were bone, liver, and mediastinum. Bone was the most common metastatic organ in Crohn's disease-associated adenocarcinoma within anorectal fistulae (six cases, 50\%), and the others were liver, lung, brain, adrenal gland, kidney, spleen, and pancreas. There was no significant difference in the dissemination and local recurrence rates between usual adenocarcinoma within anorectal fistulae and Crohn's disease-associated adenocarcinoma within anorectal fistulae.

\section{Prognostic factors}

To examine independent prognostic factors in patients with adenocarcinoma within anorectal fistulae, univariate and multivariate analyses were applied for overall survival. Age (comparing patients older than 50 years with those younger than 50 years), histological type (comparing high grade with low grade), lymphatic invasion, vascular invasion, p53 expression, and MUC5AC expression demonstrated significant associations with overall survival. Furthermore, multivariate analysis revealed that vascular invasion was the strongest significant independent predictor of overall survival in patients with adenocarcinoma within anorectal fistulae.

\section{Discussion}

We analyzed 82 consecutive cases with adenocarcinoma within anorectal fistulae by dividing them into usual adenocarcinoma within anorectal fistulae and Crohn's diseaseassociated adenocarcinoma within anorectal fistulae groups. These 2 groups of adenocarcinoma within anorectal fistulae were found to have different clinicopathological characteristics. Factors with a significant difference between both adenocarcinoma within anorectal fistulae types were age at diagnosis, macroscopic type, histological type, tendency for lymphovascular invasion, immunohistochemical expression of E-cadherin and p53, and prognosis.

Patients with Crohn's disease-associated adenocarcinoma within anorectal fistulae were approximately 20 years younger on average at the diagnosis of cancer than patients with usual adenocarcinoma within anorectal fistulae. Examining precancerous anal fistulae in adenocarcinoma within anorectal fistulae reveals that the difference in the age of cancer diagnosis probably results from the age of anal fistula onset; this is because the age at the diagnosis of anal fistula was also significantly younger in Crohn's disease-associated adenocarcinoma within anorectal fistulae than in usual adenocarcinoma within anorectal fistulae (usual adenocarcinoma within anorectal fistulae: 48.7 years on average, Crohn's disease-associated adenocarcinoma within anorectal fistulae: 31.6 years on average; data not 
shown), and there was no significant difference between the adenocarcinoma within anorectal fistulae types in the duration of anal fistulae.

The macroscopic appearance of adenocarcinoma within anorectal fistulae tended to be different from that of conventional colorectal adenocarcinoma because of differences in their origin. Adenocarcinoma within anorectal fistulae originates from the epithelium of the extraluminal anal fistula, while colorectal cancer originates from the luminal surface epithelium. According to our macroscopic classification, a large portion of the usual adenocarcinoma within anorectal fistulae cases were the endophytic or exophytic, while most of the Crohn's disease-associated adenocarcinoma within anorectal fistulae cases were the ulcerative type. The reason for the frequency of the ulcerative type in Crohn's disease-associated adenocarcinoma within anorectal fistulae could be that anal fistulae in Crohn's disease patients typically have multiple external openings [10] and invasion of adenocarcinoma along the fistulae would lead to the destruction of the anal canal structure at an early stage. Differences in the macroscopic type between usual adenocarcinoma within anorectal fistulae and Crohn's diseaseassociated adenocarcinoma within anorectal fistulae may reflect the differences in pre-existing anal fistula type and the tumor characteristics between the two types of adenocarcinoma within anorectal fistulae.

The main histological type of adenocarcinoma within anorectal fistulae was mucinous carcinoma, as reported to date [11], and our case collection reveals that high-grade mucinous carcinoma was common in Crohn's diseaseassociated adenocarcinoma within anorectal fistulae. In usual colorectal cancer, the rate of mucinous carcinoma is $11.1 \%$ and that of high-grade mucinous carcinoma is only $1.1 \%$ [12]. Colorectal mucinous carcinoma tends to arise in the right portion of colon and is usually associated with microsatellite instability [13]. In appendiceal cancer, mucinous carcinoma accounts for most cases of adenocarcinoma within anorectal fistulae, but high-grade mucinous carcinoma is very rare [14].

The overexpression rate of $\mathrm{p} 53$ in colorectal carcinomas is different between the different types, such as hereditary non-polyposis colorectal cancer, familial adenomatous polyposis-associated colorectal cancer, and ulcerative colitis-associated colorectal cancer [15-17]. In Crohn's disease-associated adenocarcinoma within anorectal fistulae, the overexpression rate of p53 was more than double that of usual adenocarcinoma within anorectal fistulae. Thus, there is a possibility that the oncogenic pathway in adenocarcinoma within anorectal fistulae can be affected by the presence or absence of Crohn's disease.

Loss of E-cadherin, which is a cell-cell adhesion molecule, is associated with poor tumor differentiation and with lymphovascular invasion in colorectal cancer [18, 19].
Furthermore, in adenocarcinoma within anorectal fistulae, the same correlation between lower E-cadherin expression and poor tumor differentiation and between lower Ecadherin expression and lymphovascular invasion was observed (tumor differentiation: $p=0.001$, vascular invasion: $p=0.0010$, lymphatic invasion: $p=0.016$ ). High rates of decreased E-cadherin expression in Crohn's disease-associated adenocarcinoma within anorectal fistulae are thought to be the result of high rates of cases with poor tumor differentiation and lymphovascular invasion in Crohn's disease-associated adenocarcinoma within anorectal fistulae.

MUC5AC is a gastric type mucin [20]. Aberrant MUC5AC expression is observed in 20-30\% of colorectal adenocarcinoma cases and is associated with a favorable outcome [21, 22]. The rate of aberrant MUC5AC expression in Crohn's disease-associated adenocarcinoma within anorectal fistulae was almost the same as that of colorectal adenocarcinoma, but was higher in usual adenocarcinoma within anorectal fistulae versus Crohn's disease-associated adenocarcinoma within anorectal fistulae.

Patients with Crohn's disease-associated adenocarcinoma within anorectal fistulae had a significantly poorer prognosis than did those with usual adenocarcinoma within anorectal fistulae. Crohn's disease-associated adenocarcinoma within anorectal fistulae cases were more commonly at an advanced stage than usual adenocarcinoma within anorectal fistulae, which reflected the poorer prognosis of Crohn's disease-associated adenocarcinoma within anorectal fistulae in all stages. However, even in the cases with the same stage (stage II), the prognosis was still poorer in the Crohn's disease-associated adenocarcinoma within anorectal fistulae than in the usual adenocarcinoma within anorectal fistulae group. One of the reasons for the poor prognosis in Crohn's disease-associated adenocarcinoma within anorectal fistulae could be its high tendency toward hematogenous metastasis. Multivariate analysis for overall survival revealed that vascular invasion was the strongest independent predictor of overall survival and the rate of vascular invasion was significantly higher in Crohn's disease-associated adenocarcinoma within anorectal fistulae than in usual adenocarcinoma within anorectal fistulae. The incidence of other organ metastases in Crohn's diseaseassociated adenocarcinoma within anorectal fistulae was more than $50 \%$ and much higher than that of usual adenocarcinoma within anorectal fistulae, while there was no difference in the incidence of local recurrence and lymph node metastasis between the two types of adenocarcinoma within anorectal fistulae. Briefly, the high potential for vascular invasion in Crohn's disease-associated adenocarcinoma within anorectal fistulae can cause hematogenous metastasis and a poor prognosis. Moreover, comparing the 5-year survival rate of stage II usual adenocarcinoma within 
anorectal fistulae with that of colorectal carcinoma showed that even usual adenocarcinoma within anorectal fistulae had a poorer prognosis than colorectal carcinoma (usual adenocarcinoma within anorectal fistulae; $71 \%$, colorectal carcinoma; $90.8 \%$ ). Thus, adenocarcinoma within anorectal fistulae is an entirely different disease entity, which has a poor prognosis compared with colorectal carcinoma.

Several characteristics were common between usual adenocarcinoma within anorectal fistulae and Crohn's disease-associated adenocarcinoma within anorectal fistulae. By comparing the sex ratio of adenocarcinoma within anorectal fistulae with that of noncancerous anal fistulae, it was revealed that men were more likely to experience malignant transformation. The sex ratio of male to female in usual adenocarcinoma within anorectal fistulae was found to be 29 , which was more than five times larger than the reported sex ratio of noncancerous anorectal fistulae [23, 24]. The male:female sex ratio of Crohn's diseaseassociated adenocarcinoma within anorectal fistulae was 2.8, whereas anal fistulae associated with Crohn's disease but without cancer were reported to have a slight female predilection [10]. Male predilection is also observed in rectal cancer arising adjacent to the site of adenocarcinoma within anorectal fistulae, although the reason for this is unknown [25].

All cases of adenocarcinoma within anorectal fistulae were confirmed as advanced carcinoma invading beyond the muscle layer. We speculate that the reason for the advanced stage at diagnosis is because of the unique primary site in adenocarcinoma within anorectal fistulae. Most anal fistulae in which adenocarcinoma within anorectal fistulae arises penetrate the muscle layer; therefore, adenocarcinoma within anorectal fistulae tends to occur deep in the anal canal wall. Moreover, until cancer has reached an advanced stage, it cannot be observed from the mucosal surface using endoscopic examination. In colorectal carcinoma, elevation of serum CEA and CA19-9 is correlated with tumor advancement [26]. Because of the advancement of adenocarcinoma within anorectal fistulae, the preoperative serum level of either CEA or CA19-9 was shown to be elevated in $70 \%$ of all patients with adenocarcinoma within anorectal fistulae. An occult blood test has low sensitivity for anorectal fistulae without carcinoma [27], and is therefore not considered useful for patients with the flat type of adenocarcinoma within anorectal fistulae. Accordingly, measuring serum CEA and CA19-9 could be practical to detect adenocarcinoma within anorectal fistulae.

Nowadays, molecular genetic profiling of cancer has become increasingly important for proper diagnosis, prognostication, and treatment. The genetic profile of adenocarcinoma within anorectal fistulae has seldom been reported and was not examined in the present study.
However, the results reported in this study highlight the necessity of launching such an investigation.

The present study is first to describe the differences between usual adenocarcinoma within anorectal fistulae and Crohn's disease-associated adenocarcinoma within anorectal fistulae through the detailed examination of 82 cases of adenocarcinoma within anorectal fistulae. Usual adenocarcinoma within anorectal fistulae and Crohn's diseaseassociated adenocarcinoma within anorectal fistulae have been found to have significant differences in clinicopathological characteristics, such as age of onset, macroscopic type, histological type, tendency toward lymphovascular invasion, immunohistochemical expression of some markers, and prognosis. In conclusion, due to these distinct differences, usual adenocarcinoma within anorectal fistulae and Crohn's disease-associated adenocarcinoma within anorectal fistulae should be considered separate clinical entities. A further study on adenocarcinoma within anorectal fistulae to categorize the disease into usual adenocarcinoma within anorectal fistulae and Crohn's diseaseassociated adenocarcinoma within anorectal fistulae is needed to improve methods of early detection and to develop better treatment.

Acknowledgements We thank Yusuke Inomori for assistance in performing the immunohistochemical examinations.

\section{Compliance with ethical standards}

Conflict of interest The authors declare that they have no conflict of interest.

\section{References}

1. Welton ML, Lambert R, Bosman FT. Tumors of the anal canal. In: Bosman FT, Carneiro F, Hruban RH, et al., editors. WHO classification of tumours of the digestive system, 4th edn. Lyon: International Agency for Research on Cancer 2010. pp. 185-93.

2. Seya T, Tanaka N, Shinji S, et al. Squamous cell carcinoma arising from recurrent anal fistula. J Nippon Med Sch. 2007;74:319-24.

3. Ky A, Sohn N, Weinstein MA, et al. Carcinoma Arising in Anorectal Fistulas of Crohn's Disease. Dis Colon Rectum. 1998;41:992-6.

4. Iesalnieks I, Gaertner WB, Glass H, et al. Fistula-associated anal adenocarcinoma in Crohn's disease. Inflamm Bowel Dis. 2010;16:1643-8.

5. Sobin L, Gospodarowicz M, Wittekind WC. TNM classification of malignant tumors, 7th edn. New York: Wiley, 2009. pp. 243-6.

6. Nishigami T, Kataoka TR, Ikeuchi H, et al. Adenocarcinomas associated with perianal fistulae in Crohn's disease have a rectal, not an anal, immunophenotype. Pathology. 2011;43:36-9.

7. Lenz HJ, Danenberg KD, Leichman CG, et al. p53 and thymidylate synthase expression in untreated stage II colon cancer: associations with recurrence, survival, and site. Clin Cancer Res. 1998;4:1227-34. 
8. Kikuyama S, Kubota T, Shimizu K, et al. Ki-67 antigen expression in relation to clinicopathological variables and prognosis in gastric cancer. Oncol Rep. 1998;5:867-70.

9. Zhang X, Yang M, Shi H, et al. Reduced E-cadherin facilitates renal cell carcinoma progression by WNT/ $\beta$-catenin signaling activation. Oncotarget. 2017;8:19566-76.

10. Coremans G, Dockx S, Wyndaele J, et al. Do anal fistulas in Crohn's disease behave differently and defy Goodsall's rule more frequently than fistulas that are cryptoglandular in origin? Am J Gastroenterol. 2003;98:2732-5.

11. Gaertner WB, Hagerman GF, Finne CO, et al. Fistula-associated anal adenocarcinoma: good results with aggressive therapy. Dis Colon Rectum. 2008;59:1061-7.

12. Secco GB, Fardelli R, Campora E, et al. Primary mucinous adenocarcinomas and signet-ring cell carcinomas of colon and rectum. Oncology. 1994;51:30-4.

13. Verhulst J, Ferdinande L, Demetter P, et al. Mucinous subtype as prognostic factor in colorectal cancer: a systematic review and meta-analysis. J Clin Pathol. 2012;65:381-8.

14. Marmor S, Portschy PR, Tuttle TM, et al. The rise in appendiceal cancer incidence: 2000-9. J Gastrointest Surg. 2015; 19:743-50.

15. Boland CR, Goel A. Microsatellite Instability in colorectal cancer. Gastroenterology. 2010;138:2073-87.

16. Lakatos PL, Lakatos L. Risk for colorectal cancer in ulcerative colitis: changes, causes and management strategies. World J Gastroenterol. 2008;14:3937-47.

17. Levi E, Stryker SJ, Rao MS. p53 Protein overexpression in colorectal tumors from patients with familial adenomatous polyposis: is it an early or late event? Am J Gastroenterol. 1996;91:11-4.
18. Dorudi S, Sheffield JP, Poulsom R, et al. E-cadherin expression in colorectal cancer: an immunocytochemical and in situ hybridization study. Am J Pathol. 1993;142:981-6.

19. Tsanou E, Peschos D, Batistatou A, et al. The E-cadherin adhesion molecule and colorectal cancer. A global literature approach. Anticancer Res. 2008;28:3815-26.

20. Lee HS, Lee HK, Kim HS, et al. MUC1, MUC2, MUC5AC, and MUC6 expressions in gastric carcinomas: their roles as prognostic indicators. Cancer. 2001;92:1427-34.

21. Walsh MD, Clendenning M, Williamson E, et al. Expression of MUC2, MUC5AC, MUC5B, and MUC6 mucins in colorectal cancers and their association with the $\mathrm{CpG}$ island methylator phenotype. Mod Pathol. 2013;26:1642-56.

22. Betge J, Schneider NI, Harbaum L, et al. MUC1, MUC2, MUC5AC, and MUC6 in colorectal cancer: expression profiles and clinical significance. Virchows Arch. 2016;469:255-65.

23. Ramanujam PS, Prasad ML, Abcarian H, et al. Perianal abscesses and fistulas - A study of 1023 patients. Dis Colon Rectum. 1984;27:593-7.

24. Isbister WH. A simple method for the management of anorectal abscess. Aust N Z J Surg. 1987;57:771-4.

25. Gao RN, Neutel CI, Wai E. Gender differences in colorectal cancer incidence, mortality, hospitalizations and surgical procedures in Canada. J Public Health. 2008;30:194-201.

26. Kim SB, Fernandes LC, Saad SS, et al. Assessment of the value of preoperative serum levels of CA 242 and CEA in the staging and postoperative survival of colorectal adenocarcinoma patients. Int $\mathbf{J}$ Biol Markers. 2003;18:182-7.

27. Sasaki K, Kunimoto M, Hirata K. Relationship between the fecal occult blood test and benign anal disorders. Hepatogastroenterology. 2001;48:445-7. 